\title{
What is the benefit of computer-assisted image analysis of liver fibrosis area?
}

\author{
Kentaro Yoshioka
}

Received: 28 November 2012/ Accepted: 29 November 2012/Published online: 15 December 2012

(C) Springer Japan 2012

Liver fibrosis is usually semiquantitatively assessed in liver biopsy specimens by the numerical system of Scheuer [1], the Metavir group [2], or Ishak [3]. Fibrosis is staged as F0, no fibrosis; F1, portal fibrosis without septa; F2, portal fibrosis and few septa; F3, numerous septa without cirrhosis; and F4, cirrhosis. Staging mainly depends on the degree of the architectural changes of liver structure.

Computer-assisted image analysis of the stained fibrosis area in liver biopsy specimens is a method for quantitatively measuring the amount of liver fibrosis [4]. It is not used for the clinical assessment of liver fibrosis in general, but is often used in the assessment of fibrosis in animal models. Its low popularity in clinical practice may be attributed to the complexity of the method.

The fibrosis stage as determined by the numerical systems and the relative area of fibrosis measured by computer-assisted image analysis usually correlate well to each other. However, discrepancy between the two sometimes occurs. Which of the two is more useful in clinical practice may depend on the objectives of assessing liver fibrosis.

The current study by Isgro et al. showed that collagen proportionate area (CPA) has a better relationship with liver stiffness measurement (LSM) and with hepatic venous pressure gradient (HVPG) compared with the Ishak stage. They also reported that CPA at 1-year post-transplantation in hepatitis $\mathrm{C}$ virus-infected patients predicts subsequent clinical decompensation more accurately than Ishak stage

This comment refers to the article available at doi:10.1007/s00535-012-0694-9.

K. Yoshioka $(\square)$

Department of Liver, Biliary Tract and Pancreas Diseases, Fujita Health University, Toyoake, Aichi, Japan

e-mail: kyoshiok@fujita-hu.ac.jp or HVPG [5]. They conclude that CPA should be the histological parameter with which to compare LSM and other non-invasive fibrosis markers and also be used to subclassify cirrhosis.

Nitta et al. [6] also reported the good correlation between LSM and fibrosis area measured by image analysis in the patients with chronic hepatitis C, while LSM and Metavir score yielded better correlation. Xie et al. [7] reported that fibrosis area measured by image analysis significantly correlated with model for end-stage liver disease score, serum bilirubin levels and prothrombin time in the patients with hepatitis B virus-related decompensated cirrhosis.

Arima et al. [8] reported that $42 \%$ of chronic hepatitis C patients with pretreatment F3-4 who obtained sustained virological response by interferon (IFN) therapy had decreased fibrosis assessed by the numerical staging system, while the fibrosis area measured by image analysis decreased in $92 \%$. Thus the computer-assisted image analysis of liver fibrosis is more sensitive to measure the reduction of liver fibrosis after IFN treatment than the numerical system.

In conclusion, the relative fibrosis area measured by computer-assisted image analysis is suitable for the comparison with newly developing non-invasive methods for fibrosis assessment, such as LSM. It is also useful to assess the degree of severe fibrosis in cirrhosis for predicting prognosis and to assess the change of fibrosis after antiviral treatment or in natural courses. It is better to add computer-assisted image analysis to the interpretation of liver biopsy in order to obtain valuable quantitative information in the specimens. The standardization and simplification of the method is needed in order that computer-assisted image analysis of fibrosis area will be widely used. 
Conflict of interest $\mathrm{K}$. Yoshioka received research grants from MSD K.K. and serves as a consultant to SANWA KAGAKU KENKYUSHO CO., LTD.

\section{References}

1. Scheuer PJ. Classification of chronic viral hepatitis: a need for reassessment. J Hepatol. 1991;13:372-4.

2. Intraobserver and interobserver variations in liver biopsy interpretation in patients with chronic hepatitis $\mathrm{C}$. The French METAVIR Cooperative Study Group. Hepatology 1994;20:15-20.

3. Ishak K, Baptista A, Bianchi L, Callea F, De Groote J, Gudat F, et al. Histological grading and staging of chronic hepatitis. J Hepatol. 1995;22:696-9.

4. Goodman ZD, Becker RL Jr, Pockros PJ, Afdhal NH. Progression of fibrosis in advanced chronic hepatitis $\mathrm{C}$ : evaluation by morphometric image analysis. Hepatology. 2007;45:886-94.
5. Manousou P, Dhillon AP, Isgro G, Calvaruso V, Luong TV, Tsochatzis E, et al. Digital image analysis of liver collagen predicts clinical outcome of recurrent hepatitis $\mathrm{C}$ virus 1 year after liver transplantation. Liver Transpl. 2011;17:178-88.

6. Nitta Y, Kawabe N, Hashimoto S, Harata M, Komura N, Kobayashi K, et al. Liver stiffness measured by transient elastography correlates with fibrosis area in liver biopsy in patients with chronic hepatitis C. Hepatol Res. 2009;39:675-84.

7. Xie SB, Ma C, Lin CS, Zhang Y, Zhu JY, Ke WM. Collagen proportionate area of liver tissue determined by digital image analysis in patients with HBV-related decompensated cirrhosis. Hepatobiliary Pancreat Dis Int. 2011;10:497-501.

8. Arima M, Terao H, Kashima K, Arita T, Nasu M, Nishizono A. Regression of liver fibrosis in cases of chronic liver disease type C: quantitative evaluation by using computed image analysis. Intern Med. 2004;43:902-10. 